\title{
Mobility management for Rpl protocol in internet of things
}

\author{
Zohreh Royaee $^{1}$, Hamid mirvaziri ${ }^{2}$, Amid Khatibi Bardsiri ${ }^{3}$ \\ ${ }^{1}$ Department of Computer Engineering, Kerman Branch, Islamic Azad University, Iran \\ ${ }^{2}$ Department of Computer Engineering, Shahid Bahonar University, Iran \\ ${ }^{3}$ Department of Computer Engineering, Bardsir Branch, Islamic Azad University, Iran
}

\begin{tabular}{l}
\hline Article Info \\
\hline Article history: \\
Received Nov 1, 2019 \\
Revised Jan 14, 2020 \\
Accepted Jan 24, 2020 \\
\hline
\end{tabular}

Keywords:

Bernoulli

Flabellum algorithm

Internet of things

Mobile nodes

\begin{abstract}
The IPv6 Routing Protocol for Low Power and Lossy Networks (RPL) was proposed for various applications of IPv6 low power wireless networks. While RPL supports various routing metrics and is designed to be suitable for wireless sensor network environments, it does not consider the mobility of nodes. Therefore, there is a need for a method that is energy efficient and that provides stable and reliable data transmission by considering the mobility of nodes in RPL networks. In this paper a new heuristic flabellum algorithm inspired by physical and biological behaviour of flabella in the sea is presented, and Flabellum_Bernoulli model based RPL Abbreviated "FBRPL"presented to avoids bottleneck and swarm problems through managing the moving nodes. flabellum algorithm consider two parameters which are node rssi and pdr and Bernoulli distribution suggested 5 steps to Initial distribution of moving nodes. Finally, the proposed algorithm's performance is evaluated using the Cooja simulator. The proposed algorithm;Flabellum_Bernoulli RPL; shows significant improvements with regards to packet delivery, and convergence and lifetime.
\end{abstract}

Copyright $(2020$ Institute of Advanced Engineering and Science. All rights reserved.

\section{Corresponding Author:}

Hamid Mirvaziri,

Department of Computer Engineering,

Shahid Bahonar University,

Kerman, Iran.

Email: hamidmirvaziri@yahoo.com

\section{INTRODUCTION}

RPL is a routing protocol devised for low power and lossy networks (LLN) that consist ofresource-limited devices. As a routing protocol that operates among IoT (Internet of Things) devices, RPL was designed to be suitable for wireless sensor network environments, and it supports variousrouting metrics to accommodate the requirements of various applications [1]. The RPL forms a wirelesssensor network using a directed acyclic graph (DAG) topology so that the cost of every node reachingthe LLN border router is minimized. Moreover, a trickle algorithm is used to maintain consistencyamong the nodes that comprise the network $[1,2]$. While such a design is optimized for IoT serviceswhere the network topology does not vary, it is difficult to maintain the routing paths in a stablemanner in an IoT service environment where the devices move frequently $[3,4]$. Therefore, consideringthat mobility is an important property of mobile devices in an IoT environment, there is a need for amechanism that is energy efficient and that provides for stable and reliable data transmission. Mobility support is a fundamental issue in RPL. Previous works on mobility support in RPLmostly aimed to achieve rapid detection of the movement of mobile nodes and the provision ofbetter links through an efficient selection of parent nodes. In other words, mobility support in RPL isdirectly related to a fast search for appropriate nodes to become the parent node when a mobile nodemakes a movement algorithms that increase or decrease the time interval for the selection of parent nodes by multiplyingor dividing a predefined interval by a certain integer value. This was based on the respective methodsproposed in each work in order to support the mobility 
of the nodes. However, mobile nodes areobjects that move in a random manner in a network. Therefore, the methods proposed by previousworks can lead to losses in the intervals during which the mobile nodes select the parent node in anRPL network. This loss may cause the increase of the packet loss rate and energy consumption whilethe information is delivered. Hence, considering these characteristics of mobile nodes, the intervalduring that a mobile node selects a parent node must be allocated dynamically based on the speed anddirection of the movement of the node. This paper proposes an algorithm for the timely transmission of solicitation messages for nodemobility support in IoT environments that require the movement of the nodes or things. The proposedalgorithm provides mobility support by dynamically adjusting the transmission period of the messagesfor selecting the parent node. This is based on the speed and direction of the movement of a mobile node.

\section{RELATED WORK}

Several researchers worked on the problem of mobile nodes in WSNs. They proposed some algorithms in order to improve some routing metrics such as overhead, latency, packet delivery ratio and network lifetime. The majority of these works are based on the modification of the RPL routing protocol because it has become a standard since 2012 [1]. In [3], the authors introduced a new protocol for mobile wireless sensor networks based on RPL and called "DMR". This protocol uses two routing metrics: the rank information and the link quality identification (LQI). The authors also added redundancy in the network in order to help mobile nodes to find easier alternative paths. In order to solve the problem of disconnection, they proposed the addition of target nodes in the routing table when there are no other nodes that have detected breaks in links. However, these two strategies of local and global repair have been already proposed in RPL protocol. In addition to that, the authors by evaluating the performance of DMR, they did not compare their strategy with native RPL. They only compared it with AODV [4] and AOMDV [5] protocols. According to the authors of papers [6,7], two constraints must be taken into consideration to adapt the RPL protocol for Vehicular Ad-hoc Networks (VANET). The first one concerns the rank and the second one is related to the loss of connectivity. Since RPL is designed for static WSNs, the rank is not updated in a timely fashion to reflect frequent changes in topology. Slow reactivity to topology changes results in a suboptimal path to the destination. Moreover, a node may loose connection of roadside infrastructure and connects to a node among its neighbors as a new parent which can lead to a loop. The authors improved the responsiveness of the protocol by introducing a strategy of immediate ETX Probing for a new neighbor and a mechanism of immediate generation of control messages (DIO, DAO) upon new parent election. They also proposed a method for detection and elimination of loops. However, their algorithm is suitable only in the case of VANETs with high node speeds. In [8], a new ME-RPL approach is proposed. It relies on the identification of mobile nodes to improve the behaviour of RPL in the case of mobility. To identify mobile nodes, the nodes publish their mobility status in the control messages. When its selected parent is changed, a node will prefer to choose a fixed node as a parent. Finally, a node will solicit its neighbourhood quickly by sending DIS control messages. Moreover, the authors modified the choice of the preferred parent. If two nodes are fixed or mobile, a node chooses the node with the lowest rank as it preferred parent. If one of the nodes is fixed and the other is mobile with different ranks then the fixed node is chosen as it preferred parent. The main drawback of this protocol is it does not respect the standard in the control messages. In addition to that, the selection of the preferred parent is not based on routing metrics. In [9], the authors proposed to use the geographical information as a new routing metric for RPL protocol in order to support frequent changes of the topology. They modified the trickle timer algorithm and proposed a strategy to adjust DIO period according to the node velocity. They compared the performance of the new approach with those of the RPL. The simulations showed that their approach is better than RPL in terms of delay, packet delivery ratio and overhead. However, the new algorithm can be applied only on VANETs since the exact movement of the mobile nodes can not always be known in advance. In the work described in [10], the authors proposed a CO-RPL approach as an extension of RPL. It relies on the mechanism of Corona. This mechanism divides the network into circular areas around the root of the Directed Acyclic Graph (DAG) called Coronas. This architecture makes the nodes quickly find an alternative parent. The authors also improved the local and global repair of the network since these mechanisms have some limitations for mobile nodes [11]. When a mobile node can not transmit data packet, it forwards it to any node in a high corona and informs its sub-DAG to stop sending the data by transmitting DIS messages. The path recovery mechanism guarantees the prevention of dropping packet at mobile node and its children. The simulations showed that the new approach improves RPL in terms of delay, energy consumption and packet loss rate. However, the authors did not address in their study the case of an hybrid network composed of fixed and mobile nodes. To sum up, some works deal with the problem of mobile nodes in the case of VANETs where nodes move in one direction and their speed are very high. 
Other works deal with the problem of mobility in WSNs but not in an hybrid environment where mobile and static nodes coexist together. Moreover, the works that have treated the problem of hybrid sensor networks did not respect the standard in both messages exchanged and preferred parent selection. This work proposes some modifications to RPL which respect the standard defined by the Internet Engineering Task Force (IETF). Authors in [12] proposed a new cost metric combining the number of hops, RSSI values, and the summation of delay to enhance RPL mobility. In addition, They define the movement notification for the mobile node to activate mobile detection and parent selection processes. Authors in [13] proposed heuristic based energy efficient communication approch hasapplied. A new development in the Genetic algorithm has presented and called asDominant Genetic algorithm to determine the optimum energy efficient routing path between sensor nodes and to define the optimal energy efficient trajectory for mobile data gathering node.

In [14] A novel approach of ant colony optimization (ACO) algorithm for discovering the optimum route for information transmission in the WSNs is proposed here for optimization and enhancement. The issue of path selection to reach the nodes and vital correspondence parameters, for example, the versatility of nodes, their constrained vitality, the node residual energy and route length are considered. Utilizing the novel technique and considering both the node mobility and the existing energy of the nodes, an optimal route and best cost from the originating node to the target node can be detected.

In [15] A mobility model attempts to mimic the movement of real mobile nodes that change the speed and direction with time. The mobility model that accurately represents the characteristics of the mobile nodes in an ad hoc network is the key to examine whether a given protocol. The aim of this paper is to compare the performance of four different mobility models.

Authors in [16] proposed temporarily evaluation the concepts, features, protection, and privacy applications of Internet of Things authorized mobile edge computing with its data protection view in our data-driven globe. In [17] Authors optimized RPL objective function by combining several RPL parameters, such as (i) Expected Transmission Count (ETX), (ii) number of hops, and (iii) average Received Signal Strength Indicator (RSSI) as inputs in a fuzzy logic model. These parameters are more influenced in mobile applications.

In [18] introduced Kalman positioning RPL (KP-RPL), a novel routing strategy for WSNs with both static and mobile nodes, based on RPL. They considered the original RPL for the communication among static nodes and position-based routing for mobile nodes, which use a novel RPL metric that combines Kalman positioning and blacklisting.

Authors in [19] presented the design of Co-RPL, an extension to RPL based on the corona mechanism that supports mobility in order to overcome the problem of slow reactivity to frequent topology changes and thus providing a better quality of service mainly in dynamic networks application.

Authors in [20] A new Modes Of Operation (MOP) is introduced to indicate the critical resource condition of the node. The required routing memory and the expected routing lifetime are proposed to determine the MOP of the node. The MOP downgrade and the MOP upgrade are introduced to address traffic congestion caused by the isolated higher resource node and to exploit the renewed resources and the resource requirement relaxation, respectively. The queue utilization based data transmission distributes data packets for load balance and network performance improvement.

\section{METHODS/EXPERIMENTAL}

According to the random structure of the position of nodes in the proposed RPL, network congestion is undeniable, since differing number of nodes request from other network nodes. In other words, each parent node may receive an unpredictable number of requests from its children. This will lead to increased traffic and as a result, create congestion in the network's nodes. In this regard, an approach for using moving nodes has been proposed in this study, in which a number of nodes will be GW or gate nodes. These nodes have a higher radio range and energy than other nodes in the network, and will use a moving unit in the network. These nodes enter the network through the working nodes process and if necessary, assume the role of parent nodes for high congestion children. In order for moving and normal nodes to cooperate in the network, changes should occur in the network's graph structure, which will be explained.

\subsection{Prioritizing and managing the moving nodes using the presented flabellum algorithm}

Being inspired by a phenomenon and exploiting special knowledge provided by the problem, heuristic algorithms explore the complicated optimisation problem space and offer a sufficiently good (optimum) solution. In the present research, given the biological and physical behaviour of flabellum's movement in an artificial system, optimisation algorithm in continuous space is presented. The physics of movement, group subset behaviour, and flabella's death indicate that these organisms are intelligent 
and attempt to hunt for a prey and survive while interacting with each other. Intelligence enables them to efficiently use the water flow and windflaw to reach their target (formation of group subset behaviour).

Typically, flabellum optimisation algorithm is implemented in two general steps:

a) Forming an artificial system with continuous time in the problem space; initial positioning of agents; determining the fit of toxins; and specifying the strategy of moving with wind power and water flow.

b) Updating the movement and parameters during algorithm implementation phases

At the beginning of any heuristic optimization algorithm, the problem space is defined. The problem space is a multidimensional coordinate system in which searching for an optimum solution occurs. In flabellum optimization algorithm, the ocean surface is considered as the problem space on which search agents (a group of flabella) are placed. Each agent in the problem space has the following features:

a) The position of each flabellum with the sensing radius

b) Movement with windflaw and water flow

c) Flabella's amount of toxins (the rate of fit)

The position of each agent in the search space indicates a solution of the optimization problem. All positions in the problem space have the neighborhood sensing radius communication medium. The competency of each agent depends on its location on the target function.

Global optimization strategy in the problem space is such that the best location found by the search agent on the target function is regarded as the global optimum. The purpose of this strategy is to describe the ocean shore so as to direct the search agents towards that path by the wind power.

While search agents are directed towards the global optimum in all states, the agents' movement during the occurrence of three states to form a group behavior is considered as the local optimum controlled by water flow and organism's swim. If we consider the system as a group of flabella, in which a position is indicative of a point in the optimization problem space, then $d$ denotes the position of the dimension and $x_{i}^{d}$ the agents.

$$
X_{i}=\left(x_{i}^{l}, \ldots x_{i}^{d}, \ldots, x_{i}^{n}\right)
$$

Once the position of each agent $(x(t))$ is randomly determined on the problem space, the agents' rate of fit (the concentration of toxins) $f_{i}(t)$ is evaluated based on their location on the problem space. In order for an agent to change its current location to a new one ${ }^{x(t+1)}$, it requires a velocity vector. The velocity vector of an agent changes from the position $V(t)$ to the next position $V(t+1)$ by wind and water powers.

$$
\mathrm{F}(t+1)_{i}^{d}=\alpha_{i}(t) \times V(t) \times \operatorname{rand}\left(G_{\text {best }}-X_{i}(t)\right.
$$

where $V$ is wind power whose constant value is $2(\mathrm{v}=2)$, and $\alpha$ is the effectiveness coefficient of the agent $i$ by wind power, which can be adjusted in the range ${ }^{[0.1<\alpha<0.9]}$ proportional to the amount of water power $V$, but is always considered constant. Rand is a random number with uniform distribution in the range $[0,1]$. G_best is the best location found by an agent.

In response to the three occurrences, the local optimum, combined with the global optimum, completes the movement strategy of the next step. In this state of the system, the water power is imposed on the agent $i$ as $\vec{F}$ (wind) $)_{i}^{d}(t)$ at time $t$ to the dimension $d$ towards the local optimum (formation of subset behaviour) in three states.

The first state: If there is a neighbourhood in the sensing radius, whose fit is the best compared to the current agent $i$, it moves one step towards that neighbourhood, and this power is calculated as follows.

$$
\mathrm{F}(t+1)_{i}^{d}=\beta_{i}(t) \times U(t) \times \operatorname{Sin}(\text { rand })\left(L_{\text {nigberhood }_{\text {best }_{i}}}-X_{i}(t)\right)
$$

where $U$ is water power with constant value $2(U=2)$, and $\beta$ is the effectiveness coefficient of the agent $i$ by the water power, which can be adjusted in the range [0.1< $\beta<0.9]$ proportional to the amount of water power $U$, but is always considered constant. Rand is a random number with uniform distribution in range $[0,1]$. $L_{\text {nigberhood }}$ best $_{i}$ is the best agent in terms of competency in the agents' neighbourhood.

The second state: If there is no best neighbourhood for the agent $i$ in the sensing radius, the agent moves one step towards its own personal memory, whose value in this state is calculated as follows 


$$
F_{i}^{d}(t+1)=\beta_{i}(t) \times U(t) \times \operatorname{Sin}(\text { rand })\left(L_{\text {nigberhood }_{\text {best }_{\text {mem }} i}}-X_{i}(t)\right)
$$

$L_{\text {nigberhood }}$ is the best personal memory of the agent $i$.

The third state: If the agent $i$ has no personal memory for the second state, then it randomly moves one step, whose value is calculated as follows.

$$
F_{i}^{d}(t+1)=\beta_{i}(t) \times U(t) \times \text { rand }
$$

The agent $x(t)$ with a sensing radius has evaluated the competency of its surrounding agents. If no agent in the neighbourhood of agent $x(t)$ has better competency, or if the neighbourhood of agent $x(t)$ is vacant and agent $x(t)$ has no personal memory on the other hand, then agent $x(t)$ randomly moves one step. The next step of agent $x(t)$ is towards the agent $G_{-}$best. The power that is generally imposed on the agent $i$ is the result of wind and water powers expressed as follows.

$$
\left(F_{i}^{d}(t)=\alpha_{i}(t) \times V(t) \times \operatorname{rand}\left(G_{-} b e s t\right)+\beta_{i}(t) \times U(t) \times \operatorname{rand}(\text { Lbest, } \text { Mbest, rand })\right.
$$

The best neighbourhood in the three states of occurrence (local optimum) is selected as the best according to the competency of the neighbourhood. Competency can be changed depending on the type of the optimisation problem (minimisation, maximisation) for the best neighbourhood. The best competency for the global optimum in this algorithm is considered as the highest competency among the community members. In other words, the agent with more competency, in the community distributed on the problem space, is selected as the global optimum. The global optimisation strategy of this algorithm is to direct the agent towards the shore and its death. Therefore, an agent with the highest competency is a global optimum directed towards the shore. This article presents a protocol called Flabellum_Bernoulli based RPL Abbreviated "FBRPL" to avoids bottleneck and swarm problems. Follow the below steps for each moving node to Initial distribution of moving nodes:

Step 1: Calculate Bernoulli parameters for all moving nodes and obtain the deployment assumption obtained in the last iteration of the flabellum algorithm.

Step 2: Create a list, "moving list", including all moving nodes The list is then reduced in the light of the Bernoulli parameters.

Step 3: In "moving list", select and delete the moving node with the highest Bernoulli parameter and randomly decide to suppress the moving node via a Bernoulli decision rule.

Step 4: If Bernoulli's decision suppresses the moving node, then the probability of identifying all moving nodes in the vicinity of "suppresses moving node" is calculated. In this case, the "moving list", is updated with new Bernoulli parameter values for each moving node.

- Step 5: If "moving list" is not empty, go back to step 3.

After Initial distribution of moving nodes, The flabellum algorithm starts with two parameters Rssi, Pdr.

\section{RESULT AND DISCUSSION}

The proposed network's simulation conditions are introduced in Table 1. According to Table 1, there are 180 sensor nodes distributed randomly in the simulation environment and evaluated in these tests, and the extent to which the proposed and the similar base method are successful in energy efficiency per successful send in the network, which leads to dynamic routing, will be evaluated.

Table 1. Simulation conditions

\begin{tabular}{cc}
\hline value & parameter \\
\hline 180 node & Number of nodes \\
$240 \mathrm{~m} * 240 \mathrm{~m}$ & Network environment \\
30 to 50 streams per minute & Network traffic rates \\
$30 \mathrm{~m}$ & Node radio board \\
5 joules & Primary energy of the node \\
CBR & Traffic type \\
$200 \mathrm{sec}$ & Simulation time \\
\hline
\end{tabular}

\subsection{Delivery rate test with various movement patterns}

However, the main challenge of delivery rate in RPL is in heavy traffics which can cause bottlenecks in the network and disturb its delivery rate. In this test, three movement models are selected for 
moving nodes in the network. In the first model, moving nodes move in the network based on a random pattern. In the second model, the movement pattern of moving nodes in the network is determined by tabu search algorithm, and the final pattern is to use the flabellum algorithm. The results indicate that the proposed flabellum method could yield a higher, acceptable delivery rate compared to the other two methods. Figure 1 shows The results of packet delivery rate test with various movement patterns for the base and proposed methods.

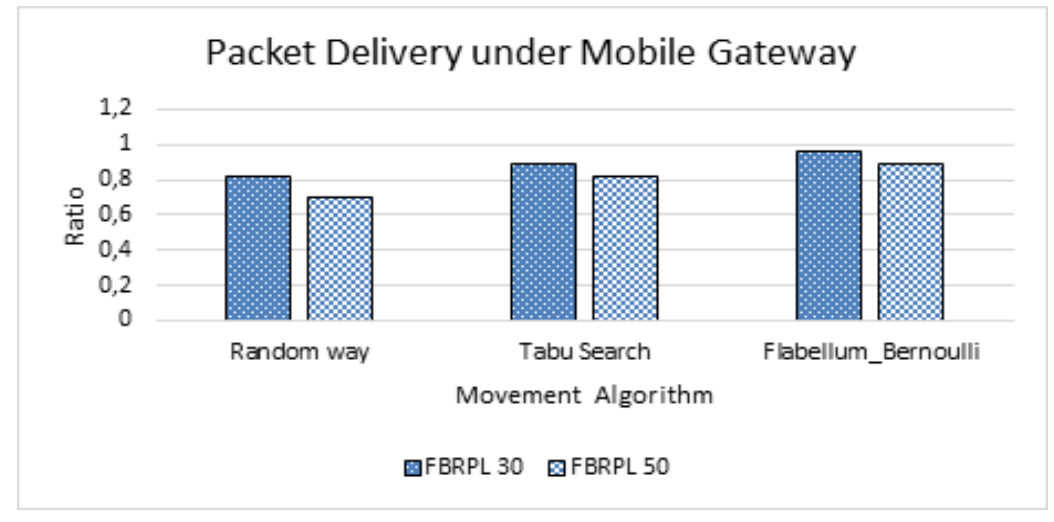

Figure 1. The results of packet delivery rate test with various movement patterns

\subsection{Network lifetime test}

The network lifetime test is performed to evaluate the network's effectiveness in saving energy for active nodes. Many studies have considered the time of death for the first and the middle nodes of the network as the main factor for network assessment. The more unbalanced the network's energy consumption, the faster this event will occur. Energy efficiency was the goal of the network proposed in this study, and an attempt was made to consider link quality and the parent's condition to prevent the premature death of the network's nodes as much as possible, and delay the death of the network's first node. Figure 2 shows the test results for two traffic criteria, 30 and 50 packets in unit of time for the base and proposed methods.

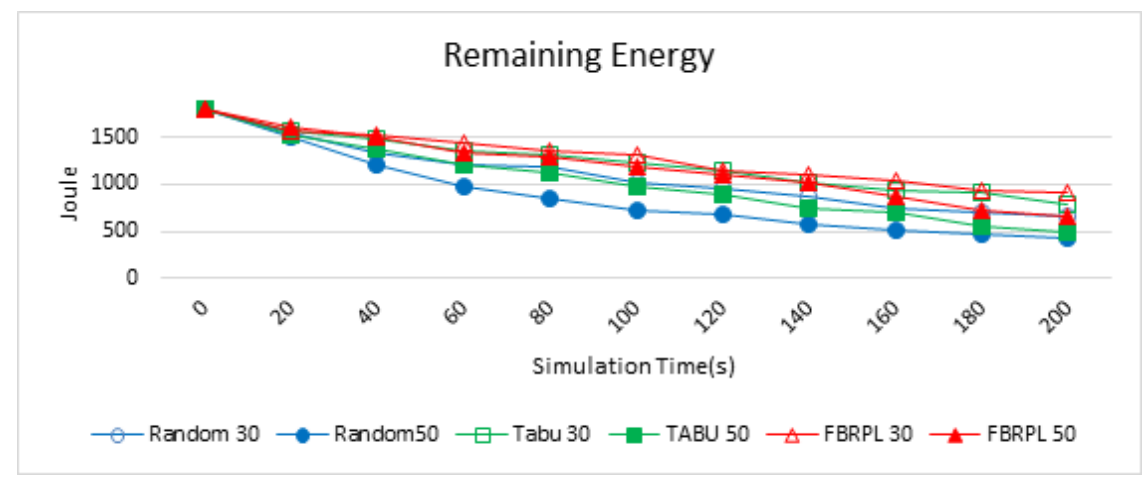

Figure 2. Network lifetime for 30 and 50 current packets in unit of time

\subsection{Scheduler reset probability test in heterogeneous network}

This parameter was included in order to study the feasibility of the proposed method to retain the current drop scheduler. The lesser the event occurs in network nodes, the lesser DIO messages are sent in the network and the more the network efficiency is increased. The use of moving nodes in stabilising network graph and creating load balance can reduce unwanted resets in drop scheduler in the network. According to the test results, flabellum movement management method has outperformed random movement and tabu search methods. Figure 3 shows the test results of drop scheduler reset rate with various movement patterns for the base and proposed methods. 


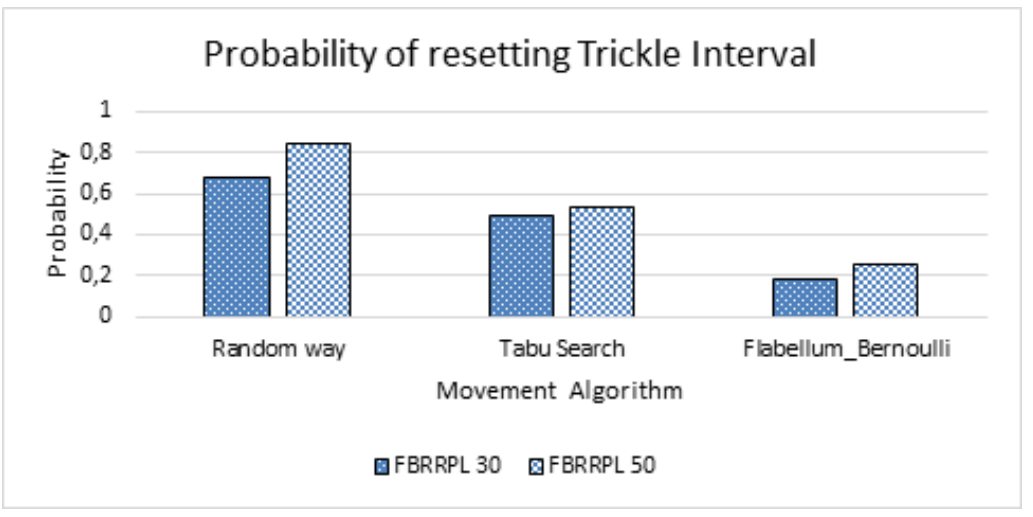

Figure 3. The test results of drop scheduler reset rate with various movement patterns

\section{CONCLUSION}

This article has evaluated the RPL routing protocol's problems under heavy and dynamic load by focusing on packet loss and network lifetime. It is discovered that the RPL standard cannot effectively manage and balance heavy loads and dynamic loads. The new heuristic flabellum algorithm inspired by physical and biological behavior of flabella in the sea was introduces, and the bottleneck and swarm problems were significantly improved by managing the moving nodes by flabellum algorithm with two parameter which are node rssi and pdr. And used Bernoulli parameters and suggested 5 steps toInitial distribution of moving nodes. After message reception is verified by the congested node, the moving node will assume the role of the congested parent node and receive messages from the other node's children and the node itself, and then transfer their messages to the node's parents. The proposed method was evaluated in Cooja in various scenarios, which proved that outperformed existing algorithms with regards to packet delivery, convergence and lifetime.

\section{REFERENCES}

[1] T. Winter, A. Brandt, J. Hui, R. Kelsey, P. Levis, K. Pister, R. Struik, J. Vasseur, and R. Alexander, "RPL: IPv6 Routing Protocol for LowPower and Lossy Networks," IETF RFC 6550, March 2012.

[2] J. Tripathi, J. de Oliveira, and J. Vasseur, "Performance Evaluation of the Routing Protocol for Low-Power and Lossy Networks (RPL), " IETF RFC 6687, October 2012.

[3] K. Hong and L. Choi, "DAG-based multipath routing for mobile sensor networks," ICTC, pp. 261-266, 2011.

[4] C. Perkins, E. Royer, and S. Das, "RFC 3561 Ad hoc On-Demand Distance Vector (AODV) Routing," Tech. Rep., 2003.

[5] M. K. Marina and S. R. Das, "Ad hoc on-demand multipath distance vector routing," Wireless communications and mobile computing, 2006.

[6] K. C. Lee, R. S. Sudhaakar, L. L. Dai, S. Addepalli, and M. Gerla, "RPL under mobility," in IEEE Consumer Communications and Networking Conference (CCNC), pp. 300-304, 2012.

[7] K. C. Lee, R. Sudhaakar, J. Ning, L. Dai, and S. Addepalli, "A comprehensive evaluation of rpl under mobility," International Journal of Vehicular Technology, pp. 1-10, 2012.

[8] I. Korbi, M. Ben Brahim, C. Adjih, and L. Saidane, "Mobility enhanced rpl for wireless sensor networks," The Third International Conference in Network of the Future (NOF), 2012.

[9] B. Tian, K. Mean Hou, H. Shi, X. Liu, X. Diao, J. Li, Y. Chen, and J.-P. Chanet, "Application of modified rpl under vanet-wsn communication architecture," International Conference on Computational and Information Sciences, 2013.

[10] O. Gaddour, A. Koubaa, R. Rangarajan, O. Cheikhrouhou, E. Tovar, and M. Abid, "Co-rpl: Rpl routing for mobile low power wireless sensor networks using corona mechanism," 9th IEEE International Symposium on Industrial Embedded Systems (SIES), pp. 200-209, 2014.

[11] O. Gaddour, A. Koubaa, S. Chaudhry, M. Tezeghdanti, R. Chaari, and M. Abid, "Simulation and performance evaluation of dag construction with rpl," Third International Conference on Communications and Networking (ComNet), pp.1-8, 2012.

[12] P.Satanasaowapak, C.Khunboa, "The improvement of node mobility in RPL to increase transmission efficiency," International Journal of Electrical and Computer Engineering (IJECE), pp. 4238-4249, 2019.

[13] D. hanthi, K. Prasanna, "Energy efficient intelligent routing in WSN using dominant genetic algorithm," International Journal of Electrical and Computer Engineering (IJECE), pp. 500-511, 2020.

[14] A.Sharmin, F. Anwar, S. Motakabber,"A novel bio-inspired routing algorithm based on ACO for WSNs," Bulletin of Electrical Engineering and Informatics, pp. 718-728, 2019 
[15] S. Laqtib1, K.Yassini, M.Lahcen Hasnaoui," Link-state QoS routing protocol under various mobility models," Indonesian Journal of Electrical Engineering and Computer Science, pp. 906-916, 2019.

[16] A.Rahman, "Mobile Edge Computing for Internet of Things (IoT): Security and Privacy Issues," IJEECS journal, vol. 18, no. 3, pp.1452-1459, 2020.

[17] I. H. Urama, H. Fotouhi, and M. M. Abdellatif, "Optimizing RPL Objective Function for Mobile Low-Power Wireless Networks," IEEE 41st Annual Computer Software and Applications Conference (COMPSAC), vol. 2, pp. 678-683, 2017.

[18] M. Barcelo, "Addressing Mobility in RPL With Position Assisted Metrics," IEEE Sensors Journal, vol. 16, no.7, pp. 2151-2161, 2016.

[19] O.Gaddour, A. Koubâa, and Mo. Abid, "Quality-of-service aware routing for static and mobile IPv6-based lowpower and lossy sensor networks using RPL,"Ad Hoc Networks, vol. 33, pp. 233-256, 2015.

[20] J. Guo, P. Orlik, and K. Ishibashi, "Resource aware hierarchical routing in heterogeneous wireless IoT networks," Eighth International Conference on Ubiquitous and Future Networks (ICUFN), pp. 599-604, 2016. 